Mélanges

de l'École française de Rome

Moyen Âge

Mélanges de l'École

française de Rome - Moyen

Âge

127-1 (2015)

Donne di Pietra. Immagini, vicende, protagoniste delle sepolture romane del

Rinascimento: una ricerca in corso - Varia - Ateliers doctoraux - Regards croisés

Christophe Masson

\title{
Faire la guerre, faire l'État. Les officiers « militaires » sous les trois premiers souverains Valois de Naples
}

\begin{abstract}
Avertissement
Le contenu de ce site relève de la législation française sur la propriété intellectuelle et est la propriété exclusive de l'éditeur.

Les œuvres figurant sur ce site peuvent être consultées et reproduites sur un support papier ou numérique sous réserve qu'elles soient strictement réservées à un usage soit personnel, soit scientifique ou pédagogique excluant toute exploitation commerciale. La reproduction devra obligatoirement mentionner l'éditeur, le nom de la revue, l'auteur et la référence du document.

Toute autre reproduction est interdite sauf accord préalable de l'éditeur, en dehors des cas prévus par la législation en vigueur en France.
\end{abstract}

\section{revues.org}

Revues.org est un portail de revues en sciences humaines et sociales développé par le Cléo, Centre pour l'édition électronique ouverte (CNRS, EHESS, UP, UAPV).

Référence électronique

Christophe Masson, «Faire la guerre, faire l'État. Les officiers « militaires » sous les trois premiers souverains Valois de Naples », Mélanges de l'École française de Rome - Moyen Âge [En ligne], 127-1 | 2015, mis en ligne le 10 février 2015, consulté le 19 février 2015. URL : http://mefrm.revues.org/2531

Éditeur : École française de Rome

http://mefrm.revues.org

http://www.revues.org

Document accessible en ligne sur :

http://mefrm.revues.org/2531

Document généré automatiquement le 19 février 2015.

(c) École française de Rome 


\section{Christophe Masson}

\section{Faire la guerre, faire l'État. Les officiers «militaires » sous les trois premiers souverains Valois de Naples}

À la suite de l'adoption de Louis I ${ }^{\mathrm{er}}$, duc d'Anjou, par Jeanne $\mathrm{I}^{\text {̀re }}$, reine de Naples, en 1380, plusieurs princes français de la maison Valois conduisirent des entreprises militaires à destination de ce royaume. Après Louis $\mathrm{I}^{\mathrm{er}}$, de 1382 à 1384 , ce fut au tour de son fils Louis II, de 1390 à 1399 et de 1409 à 1411, et de ses petits-fils Louis III, de 1419 à 1434, et René I ${ }^{\text {er }}$ de 1435 à 1442 et de 1459 à 1463 par l'intermédiaire de son fils Jean avant que, par héritage, ces prétentions n'échoient aux rois de France et ne soient le prétexte des Guerres d'Italie'. Loin de se limiter à de simples campagnes militaires conclues par des échecs, ces entreprises revêtirent l'aspect de réels projets politiques au sens le plus complet du terme. $\mathrm{Si}$, à l'époque, les institutions royales françaises se centralisent, tirant en cela parti de la volonté du roi (comme dans le cas de Charles V) ou de ses « absences » (pour prendre l'exemple de Charles VI), le royaume de Naples connaît la situation presque inverse. Face à celle-ci, les ducs d'Anjou adoptent une stratégie particulière: ils font le choix de la création d'une administration politique originale, assez éloignée du modèle français et construite selon des modalités choisies ou subies par eux, quand bien même ils n'auraient exercé leur pouvoir que pour une période limitée, voire dans une seule partie de ce royaume.

L'un des champs d'application de cette politique fut celui du contrôle des structures militaires. À côté de la justice, le pouvoir des armes constitue en effet un autre pilier indispensable à l'existence de l'État, ainsi qu'à son contrôle. Le royaume de Naples se trouvant tout au long des campagnes angevines disputé entre deux dynasties (Valois d'Anjou et Anjou-Duras dans un premier temps, Valois d'Anjou et Aragon dans un second), aucun des partis en présence ne pouvait faire l'économie d'un appareil militaire à même de lui assurer la victoire. Dépassant les limites de la seule armée de campagne, celui-ci a pour principal objectif de coordonner la gestion et la défense des places fortes, véritables remparts en même temps que soutiens indispensables du pouvoir politique. Plus encore, il doit se concevoir comme un tout protéiforme marqué par des mouvements de fond qui se manifestent aussi bien dans la constitution d'une armée que dans la maîtrise d'un important réseau de forteresses. Dans le cadre de cet article, nous nous attacherons plus précisément à l'étude de l'action des trois premiers ducs d'Anjou de la maison Valois, c'est-à-dire à ceux qui eurent à établir leur pouvoir sur le royaume concurremment ou en accord avec la dynastie de Duras, soit de 1382 à $1434^{2}$. Malgré la variété des champs d'action de cette politique, les sources sont assez rares. En premier lieu parce que, du fait même de la nature très épisodique ou partielle du pouvoir angevin sur le royaume de Naples, les documents ont, de tout temps, été particulièrement dispersés, que ce soit au Moyen Âge, ou, plus tard, aux époques modernes ou contemporaines, ce qui n'a pas été sans influer négativement sur leur conservation. En second lieu parce que, lors de la campagne d'Italie de 1943, les troupes allemandes, alors en retraite, ont délibérément incendié les archives napolitaines que l'on avait pourtant pris soin d'éloigner du théâtre des combats $^{3}$. Ce sont ainsi plusieurs siècles d'histoire qui ont disparu, au centre desquels se trouvait la majeure partie de la documentation médiévale relative au royaume. La recherche sur ces questions se révèle donc particulièrement ardue et il faut suivre bien des chemins de traverse pour parvenir à recomposer l'administration angevine du royaume de Naples, ou à tout le moins en ébaucher les contours. Il ne s'agit toutefois pas d'une tâche impossible puisque, outre les sources narratives assez nombreuses mais dont la précision est évidemment toute relative dès lors que l'on s'intéresse aux titulatures des officiers médiévaux, plusieurs dépôts d'archives étrangers au royaume ou recueils de sources diplomatiques permettent de pallier, à tout le moins partiellement, les lacunes déjà évoquées ${ }^{4}$. 
On peut, de la sorte, avoir une connaissance assez précise de la composition des armées mises sur pied par les ducs d'Anjou. À cette occasion, on observe que ces derniers respectent un modus operandi qui ne semble pas connaître de véritable changement au fil du temps. Les troupes qu'ils convoquent peuvent être divisées en cinq groupes, quantitativement et qualitativement inégaux. On rencontre, en premier lieu, une frange de l'aristocratie de l'apanage français de ces princes (duché d'Anjou, comté du Maine...). De la même façon, une fois le pouvoir des ducs établi sur la Provence - dont Louis $\mathrm{I}^{\mathrm{er}}$ hérita avec le royaume de Naples de la reine Jeanne, également maîtresse de ce comté -, les Angevins purent y recruter de nobles hommes de guerre et des officiers tout dévoués au succès de leurs nouveaux seigneurs ${ }^{5}$. En troisième lieu viennent les régnicoles, qu'ils soient, principalement, des feudataires ou, dans une moindre mesure, des bourgeois. Véritablement au centre des rivalités entre les prétendants au trône, ils n'hésitèrent pas à négocier leur fidélité et leur ralliement en fonction des caprices de la Fortune dont eurent à souffrir aussi bien les ducs Valois que les princes de Duras ${ }^{6}$. Propres à l'Italie de la fin du Moyen Âge et du début de l'époque moderne, les condottieri et leurs contingents soldés complétèrent à plus d'une reprise les rangs de tous les compétiteurs impliqués dans cette rivalité. Italiens ${ }^{7}$, Allemands ${ }^{8}$ ou Bretons ${ }^{9}$, nombreux furent ces professionnels de la guerre à apparaître dans les armées angevines. Ajoutons-y, pour finir, un groupe hétérogène composé d'hommes liés aux ducs d'Anjou par d'autres canaux, à l'image des Bretons qui suivirent Marie de Blois à la cour de son époux Louis $I^{\text {er }}$ et qui, de là, firent le voyage d'Italie.

La création d'une armée constitue très fréquemment la première étape de l'établissement d'un pouvoir politique. Les Angevins procéderont de la sorte dans le cas du royaume de Naples. C'est en effet au moment où les hommes d'armes qui les accompagneront sont choisis que les ducs d'Anjou créent, certes sur un mode embryonnaire, le futur cadre de leur appareil administratif. Même si leurs entreprises n'ont jamais abouti à l'établissement d'une domination durable sur le royaume, les mécanismes de désignation des officiers sont déjà visibles dès ce moment. On le remarque, par exemple, dans le cas du Tourangeau Pierre de Bueil qui accompagne Louis $\mathrm{I}^{\text {er10 }}$ et en reçoit la charge de maréchal des armées avant de se voir inclus dans le conseil de régence que celui-ci, mourant, organise en faveur de son fils en $1384^{11}$, dans celui du capitaine d'aventure Bernardon de Serres qui en 1398 reçoit de Louis II le capitanat de L'Aquila ou la vice-régence de Naples ${ }^{12}$ ou encore avec le Provençal Jean de La Baume à qui l'on remet en 1383 le comté de Sinopoli ${ }^{13}$, le comté étant, dans le royaume, l'une des plus importantes circonscriptions administratives en même temps que l'un des sommets de la hiérarchie nobiliaire.

Les hommes d'armes franco-provençaux ne sont pas les seuls à ainsi intégrer les rouages du second royaume angevin de Naples, certes en gestation. Comme on l'a déjà évoqué, les ducs d'Anjou bénéficient, dans le royaume, du soutien d'une partie non négligeable de l'aristocratie régnicole. Très significatif est ainsi le cas d'Antonio dalla Ratta, comte de Caserta, qui, très rapidement après le début des entreprises de Louis Ier, est fait logothète et protonotaire du royaume de Naples ${ }^{14}$, exerçant de la sorte, à tout le moins nominalement, les fonctions d'un «premier ministre » du souverain. De la même façon, Jean de Luxembourg, comte de Conversano, après avoir servi Jeanne $\mathrm{I}^{\mathrm{èr}}$ et Louis $\mathrm{I}^{\mathrm{er}}$, rejoint la troupe du fils de ce dernier qui en fait, en 1392, son lieutenant dans le royaume de Naples ${ }^{15}$. Cette habitude de concéder des responsabilités extra-militaires à des vassaux présents dans les armées se reproduit à de nombreuses reprises, comme avec Venceslao Sanseverino vice-roi de Calabre en $1391^{16}$ ou, dès avant le départ de Louis II, encore enfant, pour la Campanie en 1390, avec trois gubernatores regni Sicilie qui lui avaient été « désignés » en la personne de trois des plus puissants feudataires du royaume, à savoir Raimondo del Balzo Orsini, Tommaso et Francesco Sanseverino ${ }^{17}$.

$7 \quad$ Si l'armée n'est pas le seul lieu d'origine des futurs officiers napolitains, elle fait véritablement figure de réservoir de personnalités compétentes et de confiance pour les souverains angevins qui ont certainement pu observer les qualités de chacun au cours de leurs campagnes. Lorsque l'on peut étudier la transformation d'armées de conquête en appareil administratif - et cela est 
plus aisé, du fait de sources plus riches et variées, dans le cas des campagnes de Louis Ier et de son fils - on constate en effet que les élites que l'on installe dans le royaume sont presque uniquement des personnalités présentes dès le début de la campagne. Le choix des membres de ces armées pour exercer des responsabilités d'un autre ordre s'explique de deux façons complémentaires: d'une part du fait de la confiance que leur témoigne le prince - qui s'exprime par leur présence dans l'armée que celui-ci a composé afin de se rendre à Naples - et de l'autre du fait de la rapidité avec laquelle ils peuvent passer du statut d'homme d'armes à celui de fonctionnaire, dans la mesure où ils se trouvent sur place et sont au fait la situation locale. Quoiqu'il en soit de ces objectifs, l'échec final de ces campagnes empêcha une transformation pérenne des cadres de l'armée en agents du gouvernement angevin du royaume de Naples, les Angevins et Provençaux revenant de la Péninsule sans avoir jamais vraiment pu y faire souche, à l'inverse de ce qui eut lieu, par exemple, à l'époque des Capétiens d'Anjou. Ainsi, au lendemain du décès de Louis III à Cosenza en 1434, ceux qui l'avaient accompagné outremonts firent-ils rapidement le voyage de retour ${ }^{18}$, signe que leurs intérêts dans le royaume étaient bien moindres que ceux qu'ils étaient certains de retrouver en Anjou, en Maine ou en Provence. Mais ambitions il y eut tout de même, furent-elles au final bien déçues, ainsi que certaines informations le laissent assez clairement deviner. On comprendrait mal, sinon, la cession qui leur fut faite de fiefs prestigieux, à l'image du despotat de Morée dont est investi le beau-frère de Louis $\mathrm{I}^{\mathrm{er}}$, Henri de Blois ${ }^{19}$, du marquisat de Corfou conféré en 1385 à Foulques II d'Agoût, l'un des membres de cette prestigieuse famille provençale qui fournissait depuis longtemps des hommes de confiance aux comtes de Provence ${ }^{20}$ ou de la principauté de Capoue remise à Pierre, frère du pape Clément VII et comte de Genève ${ }^{21}$. Si des honneurs de ce genre, certes titulaires, sont concédés, ce fut pour marquer la volonté d'inscription durable des Angevins dans le royaume de Naples et, dans le cas de la Morée, dans l'empire de Constantinople dont se prévalait le duc d'Anjou depuis qu'il en avait hérité de Jacopo del Balzo, prince de Tarente, en $1383^{22}$.

Au surplus, on peut se demander si le terreau était véritablement favorable à une inscription durable de Français ou de Provençaux dans les structures militaires du royaume de Naples. Les entreprises angevines ne sont en effet pas de véritables guerres de conquête. Au moment où ils prennent le chemin de l'Italie, les ducs sont certains de trouver sur place des soutiens d'importance, et ce d'autant plus que ceux-ci furent, plus d'une fois, à l'origine de leur départ. On peut ainsi évoquer la figure majeure d'Ugo Sanseverino, comte de Potenza, qui passa plusieurs années à la cour de Provence afin d'obtenir de la duchesse, Marie de Blois, l'envoi en Italie de son fils Louis $\mathrm{II}^{23}$. La fidélité de la noblesse régnicole, et avant tout calabraise ${ }^{24}$, à la cause angevine, que ce soit contre les Duras ou face aux Aragonais, faisait peu de doutes. C'est ainsi que Giorgio Caracciolo, frère de Luigi, comte de Gerace, sert non seulement Louis III, qui lui a remis le capitanat du casale de Forleto, mais aussi, à sa suite, René d'Anjou, avant de s'avouer vaincu par Alphonse d'Aragon ${ }^{25}$. Cette fidélité se construisait non seulement sur un attachement à la dynastie d'Anjou mais aussi sur les larges bénéfices dont celle-ci pouvait les gratifier. Les en frustrer en faveur des Français ou des Provençaux «importés » n'aurait pas peu nui à cette fidélité. C'est ce qui advint par exemple lorsque Louis de Montjoye, proche du pape $^{26}$, reçut la charge de Grand Justicier du royaume, ce qui entraîna pour un temps la famille Sanseverino à rejoindre le parti Duras ${ }^{27}$. On remarque d'ailleurs qu'un processus semblable est à l'œuvre lorsque Louis Ier prend le pouvoir en Provence. Il y conserve comme officiers des fonctionnaires locaux qu'il ne remplace pas par des officiers originaires de son apanage ${ }^{28}$. Qui plus est, les expériences antérieures l'ont déjà montré, et se répéteront par la suite, le maillage réalisé par les anciens lignages régnicoles sur les fiefs comme sur les offices royaux ne peut jamais être véritablement détricoté tant sa force repose sur une multitude d'alliances familiales qui lui rend, dans la plupart des cas, vigueur presque aussi rapidement qu'il s'est trouvé déstructuré ${ }^{29}$. Au niveau de l'administration, cette situation s'illustre dans la part majoritaire de l'aristocratie dans les charges «publiques », au détriment des juristes ${ }^{30}$. Certes il était parfois nécessaire de les faire contrôler par des "étrangers », c'est-à-dire avant tout des Français ou des Provençaux, ou des hommes nouveaux. C'est ce que ne manquèrent pas de faire les 
Duras, Jeanne II désignant en 1417 Lorenzo Attendolo vice-roi dans le Capitanat et la province de Bari afin d'y affermir son pouvoir ${ }^{31}$ et son frère Ladislas remettant des fiefs retournés au fisc à des hommes de guerre qui pourraient contrebalancer les anciens féodaux, ainsi qu'il le fit par exemple avec Alberigo da Barbiano et le marquisat de Pescara ${ }^{32}$. Mais il s'agissait là d'exceptions qui ne parvenaient pas à endiguer le véritable mouvement de fond. Qui plus est, les Valois d'Anjou se montrèrent globalement peu enclins à distribuer à large échelle des offices régnicoles à des «étrangers ». Peut-être était-ce là une façon de rassurer leurs alliés sur leurs intentions et, par voie de conséquence, une façon de se distancier des pratiques de Charles III ou de Ladislas qui n'avaient pas laissé des souvenirs unanimement positifs à leur aristocratie, ce qui était encore plus vrai dans le cas du second qui, à plus d'une reprise, écrasa dans le sang les rébellions, passées ou présentes, de ses vassaux. Si le royaume de Naples devait être changé par la conquête angevine, aux yeux des Napolitains, cela ne pouvait signifier la perte de leurs privilèges.

$\mathrm{Si}$ ces privilèges furent jalousement conservés, qu'advint-il lorsque certains soutiens des Duras furent éliminés de la scène politico-administrative du royaume? Fut-ce une opportunité pour les Franco-Provençaux de s'implanter dans le royaume? Outre le cas de fidèles de Charles III ou de Ladislas qui embrassèrent le parti angevin et, de ce fait, furent confirmés dans leurs possessions, que devaient devenir les biens de ceux qui étaient vaincus sur le champ de bataille? À qui leurs « dépouilles », conquises ou à conquérir, devaient-elles revenir ${ }^{33}$ ? Le Journal du chancelier de Marie de Blois, l'évêque de Chartres Jean Le Fèvre, livre de nombreux exemples de telles redistributions. Si celles dont il nous a conservé le souvenir eurent principalement lieu à l'époque où les ducs et la duchesse d'Anjou étaient absents du royaume de Naples - résidence cisalpine du chancelier oblige -, elles n'en traduisent pas moins les ambitions des fidèles napolitains des ducs d'Anjou. On peut ainsi voir le chevalier Jacobo Zurolo, vétéran de la campagne de Louis ${ }^{\text {er }}$, bénéficier de la remise du château de « Rigniani », possession d'un rebelle ${ }^{34}$, ou l'un de ses compagnons d'armes, Corrado d'Acquaviva, comte de San Valentino, dans les Abruzzes, recevoir les biens de plusieurs Durazzistes et obtenir la confirmation des possessions de sa famille dans le royaume ${ }^{35}$. Nulle raison de penser, donc, que ces ambitions se soient tues au moment où les ducs d'Anjou combattaient les Duras les armes à la main. Niccolo de San Framondo, comte de Cerreto, est d'ailleurs gratifié de plusieurs dons par Louis Ier, dons qui lui seront confirmés par Marie de Blois en $1386^{36}$.

Les registres de Louis III laissent encore moins de place au doute. L'action de ce prince, principalement concentrée sur le duché de Calabre dont il était le titulaire au titre d'héritier adopté par la reine Jeanne II et où il savait trouver les partisans les plus déterminés, est effectivement assez bien documentée ${ }^{37}$. Alors qu'il se trouve dans la Péninsule, avant et après son adoption, le duc d'Anjou va ainsi multiplier les cessions et les dons, remettant par exemple à Nicola, déjà comte d'Arena, le comté de Stilo au cours de l'hiver 1425-1426 ${ }^{38}$. Une certaine « exportation » de fonctionnaires à destination de la Calabre est toutefois aussi à l'œuvre comme on le voit avec l'exemple de deux trajets des environs de Naples au sud de la Péninsule que firent Luigi Arcuccio, de Capri à la châtellenie des casali de Cosenza en $1423^{39}$ ou Giacomo Arcuccio, de l'île d'Ischia au capitanat d'Acri, non loin de Cosenza, six ans plus $\operatorname{tard}^{40}$. Bien qu'entouré de conseillers originaires de son apanage ou du comté de Provence, Louis n'en témoigne pas moins une sollicitude réelle aux intérêts de ses sujets napolitains, que ceux-ci soient d'importants féodaux ou des personnages de moindre statut social.

On le voit, les partisans angevins originaires du royaume faisaient valoir leurs exigences. Certes ils rejetaient le pouvoir Duras mais leur ralliement n'était pas gratuit. On les a déjà vus acquérir les biens confisqués aux « rebelles » ou prétendre à ceux que ces derniers possédaient encore. Toutefois, il y avait plus. Les rivalités entre les dynasties de Duras et de Valois leur offraient l'occasion de tirer à nouveau profit d'un pouvoir royal bien faible et, partant, bien peu en mesure de se les aliéner. C'est ainsi vers l'obtention de nouveaux offices royaux qu'ils dirigèrent une partie de leurs efforts. Il ne suffisait pas, ou plus, de s'emparer du château ou des casali appartenant à quelque seigneur voisin et d'y exercer son pouvoir personnel, sous la protection des bannières de l'un ou l'autre souverain en compétition: il importait aussi 
d'accroître son pouvoir juridictionnel en exerçant des droits royaux, que ceux-ci s'appliquent directement sur leurs propres possessions ou à la périphérie de celles-ci ${ }^{41}$. Au vu de la situation périlleuse qu'ils connaissaient depuis la fin du règne de Jeanne Ière, les souverains napolitains voyaient dans la concession de droits royaux aux comtes une façon de s'assurer de l'exercice de ces droits et donc du maintien d'une certaine autorité royale, quand bien même cela renforcerait in fine le pouvoir desdits comtes ${ }^{42}$. Dès les premiers mois de l'expédition de Louis $\mathrm{I}^{\mathrm{er}}$, le ton est donné. Rinaldo Orsini, comte de Tagliacozzo, est ainsi fait gouverneur de L'Aquila, une ville située à deux petites journées de marche de son fief principal ${ }^{43}$. Niccolo Ruffo, marquis de Crotone, parvient quant à lui à conserver ses capitanat et châtellenie de Bova, qu'il reconnaisse le pouvoir d'Alphonse V d'Aragon ou de Louis III d'Anjou ${ }^{44}$. Ce même Louis III revêt le comte de Sinopoli, Carlo Ruffo di Calabria, de l'autorité de portulan sur les côtes de ses fiefs en $1428^{45}$. Ces concessions complétaient d'une part le rôle d'officier public du comte, en augmentant ses prérogatives et donc sa capacité à établir son autorité et, à tout le moins officiellement, celle du souverain, et de l'autre représentaient pour ce même souverain une alternative à la remise de fiefs, dont le nombre était limité, à des hommes de confiance ${ }^{46}$. On constate par ailleurs que les mêmes mécanismes se retrouvent dans le chef de la petite noblesse ou de la bourgeoisie. Ainsi, Roberto di Martorano est fait le même jour capitaine de cette place de Martorano et propriétaire de tous les biens féodaux de la localité La Serra de Chance, dans ce même territoire de Martorano ${ }^{47}$ tout comme Giovanni Sersale, originaire de Sorento, reçoit les fief, châtellenie et capitainerie d'Aiello ${ }^{48}$. Chez les Calabrais, on peut citer Ladislao Busurgi, habitant de Reggio di Calabria, qui est fait garde des ports voisins situés entre Capo Stilo et Capo Vaticano ${ }^{49}$ tandis que son conbourgeois Antonio Foti devient garde des ports de Reggio $^{50}$. En somme, dans le cadre des offices militaires ${ }^{51}$, la « quête » des charges se concentre autour des titres de portulan, de châtelain ${ }^{52}$ ou de capitaine, ces deux derniers étant souvent associés $^{53}$. Le souci d'efficacité, du point de vue du souverain, et de cumul des pouvoirs, de la part de l'officier, se trouve peut-être encore mieux exprimé avec l'exemple du secrétaire Pietro di Paolo nommé à la fois trésorier, procureur du maître portulan et maître du sel de Valle di Crati et de Terra Giordana, un justiciérat de la Calabre, pour une année d'indiction à compter du 30 août $1432^{54}$.

Comme les fiefs, plusieurs de ces charges pouvaient être transmises par voie d'hérédité. On le voit par exemple en 1406 lorsque Marie d'Enghien, qui vient de perdre son époux Raimondo del Balzo Orsini, insiste auprès de Louis II afin que l'office héréditaire de capitaine et châtelain de Brindisi et Barletta soit bien remis à son fils Giovanni Antonio ${ }^{55}$. Ces deux localités se trouvant dans les possessions des Del Balzo-Enghien, on voit bien que les charges publiques sont convoitées par les feudataires dans le but d'accroître le pouvoir qu'ils détiennent déjà sur leurs seigneuries. Qui plus est, le défunt Raimondo était auparavant parvenu à se faire octroyer par Louis Ier la châtellenie de Bari, l'une des principales villes de la région sur laquelle s'étendait son pouvoir ${ }^{56}$.

En somme, qu'importe la façon dont ces offices leur étaient remis, les feudataires tout comme les officiers cherchaient à obtenir, de façon plus ou moins consciente et plus ou moins complète, leur autonomie vis-à-vis du pouvoir central. Cette autonomie, obtenue par l'intermédiaire de la concentration, en leurs seules mains, de tous les droits pouvant s'exercer sur leur territoire, devait leur permettre d'être, aux yeux de leurs sujets et de leurs pairs, voire de celui qui devait être leur seigneur, les réels maitres d'une principauté plus ou moins embryonnaire. La noblesse, habituée depuis longtemps à traiter avec les rois de Naples comme avec des «partenaires » plus qu'avec des souverains, trouva ainsi dans la rivalité ValoisDuras le terreau propice à la réalisation de ses ambitions, à savoir un arrondissement de ses possessions ou un accroissement de ses pouvoirs d'ordres administratifs, judiciaires ou militaires. Cette accumulation d'offices, de natures variées, leur assurait en effet fortune et prestige social ${ }^{57}$. Fermement établis sur leurs possessions, les grands barons bénéficiaient, visà-vis des Duras et des Valois, d'un réel ancrage local que ces princes étrangers ne pouvaient obtenir qu'en s'attachant leur sympathie. Cette relative position de force fut donc l'occasion d'obtenir des concessions en grand nombre de la part de princes qui n'avaient d'autre choix 
que de se tourner vers eux au moment de s'établir dans le royaume. Loin toutefois de n'être qu'à sens unique, l'intérêt était au contraire réel pour chacun des deux partis. Si les régnicoles voyaient leur état accru par les diverses bénéfices ainsi collectionnés, les ducs d'Anjou tout comme les Duras d'ailleurs - trouvaient en eux des chefs de guerre et des partisans expérimentés et connaisseurs du terroir où devaient se conduire leurs guerres ainsi que des informateurs avisés de la réalité politique napolitaine et, plus largement, italienne.

Dans le même temps, les officiers choisis au sein de la composante "franco-provençale » de l'armée angevine sont extrêmement rares. On retiendra, en 1383, la figure du chevalier savoyard Boniface de Challant nommé capitaine de Montesarchio - et encore le devint-il à l'instigation de son maître Amédée VI, présent au côté du duc d'Anjou lors de son voyage d'Italie ${ }^{58}$ - et, à l'époque de Louis III, les exemples d'Hélion de Glandèves qui reçoit la co-seigneurie du château de "Garda ${ }^{59}$ ou du chevalier Tristan de La Jaille, châtelain de Reggio di Calabria, possession royale, jusqu'à sa mort survenue en $1429^{60}$. Notons par ailleurs qu'il semble bien que ces officiers se soient eux-mêmes fait accompagner de compatriotes au moment d'exercer ces offices ${ }^{61}$. Lorsque Louis II compte s'établir à Tarente, c'est à Macé de Beauvau, lieutenant d'un précédent capitaine de la ville, Robert de Droys ou de Dreux ${ }^{62}$, qu'il confie la garde du château ${ }^{63}$. Sous Louis III, c'est la place de Cosenza qui est confiée à la garde de son sénéchal Gui de la Bossaye de 1432 à 1434 , soit au moment où le duc s'y est établi ${ }^{64}$. Et si Pierre de Beauvau est en charge de cette même ville de Cosenza dès l'hiver 1425-1426, alors que Louis réside encore à Aversa, en Campanie, à la cour de la reine Jeanne II, c'est parce que son seigneur a été créé, au même moment, lieutenant général du duché de Calabre ${ }^{65}$. En somme, lorsque les Franco-Provençaux reçoivent une responsabilité militaire, celle-ci est dans la majeure partie des cas située dans l'environnement immédiat du duc d'Anjou, une situation qui s'illustre avec le plus de clarté à l'époque de Louis III. C'est en effet avec ce souverain que, d'après ce que nous laissent voir les sources, les Franco-Provençaux sont le moins souvent chargés de missions les établissant loin de leur seigneur, puisqu'on les trouve avant tout en charge des postes clés de l'appareil bureaucratique, qu'il soit à caractère judiciaire, militaire ou administratif ${ }^{66}$. Peut-être sa situation politique - il est considéré par Jeanne II comme son rival puis après 1423 comme son héritier - a-t-elle amené Louis à conserver autour de lui des fidèles dont il avait moins à se méfier que les courtisans napolitains?

Quand il peut se comporter en réel souverain, c'est-à-dire avant son adoption et au moment où il gouverne la Calabre où l'a envoyé en 1428 le favori de la reine et Grand sénéchal du royaume, ser Giovanni Caracciolo, jaloux de sa popularite $e^{67}$, Louis ne change pas de comportement mais ne fait qu'amplifier son action, sans en modifier les lignes directrices. En 1422 il autorise le parlement de Cosenza à se réunir sans devoir obtenir l'accord des officiers royaux ${ }^{68}$, comme pour marquer la confiance qu'il témoignait alors à l'égard des Calabrais dans sa lutte contre les Duras. Après 1423, et avec plus de force encore à partir de son arrivée dans son duché, il s'efforce de rendre à la région le bien-être économique grâce à des interventions dans les champs administratifs, bureaucratiques et défensifs. Il renforce ainsi les places fortes, confie aux indigènes nombre de charges locales mineures et entreprend un action pacificatrice en récupérant les localités les plus à sa portée parmi celles qui s'étaient ralliées aux Aragonais ${ }^{69}$. Ainsi, ses décisions accentuent celles de ses père et grand-père plus qu'elles ne marquent une rupture avec la façon dont ils avaient eux-mêmes agi. Le royaume de Naples, loin d'être entièrement soumis à leur pouvoir, est plus associé aux possessions angevines qu'il n'y est aggloméré. Ses particularités sont respectées et son administration n'est pas modifiée en profondeur comme elle aurait pu l'être si l'objectif des princes d'Anjou avait été de se calquer sur les institutions royales françaises. Le projet de la transformer une fois le pouvoir angevin établi sans concurrence exista-t-il? On ne peut l'affirmer, même si l'exemple de la Provence, où les ducs purent imposer leurs vues à des sujets qui, au final, ne se trouvaient pas en position de les refuser ${ }^{70}$, laisse émettre l'hypothèse que ces concessions faites aux régnicoles ne devaient, à tout le moins dans l'esprit des trois Louis, n'avoir qu'un temps. Ce qui paraît clair, toutefois, c'est le choix d'une union personnelle appliquée à trois ensembles géographiques 
(apanage, Provence, Naples) qui n'ont pas, à ce moment, vocation à être fusionnés en un seul État.

Les cadres militaires du futur État angevin de Naples restent donc attachés au duc et ne le quittent qu'à de rares occasions. Présents à ses côtés lors des campagnes, à l'époque de Louis I ${ }^{\text {er }}$ et de Louis II, ou au sein même de la cour qu'un jour il est censé diriger, dans le cas de Louis III cohabitant avec Jeanne II, ils agissent comme le ferait un conseil de guerre tel que celui organisé à l'intention de Jean, comte de Nevers, à la veille de la croisade de Nicopolis ${ }^{71}$. Les princes d'Anjou ont donc avant tout cherché à ne pas s'isoler de leurs fidèles, comme lorsque Bertrand de Beauvau, l'un des membres de cette famille qui s'éleva grâce à son service ducal, fut chargé des governorat et lieutenance générale de Calabre au moment même où Louis III y résidait ${ }^{72}$. C'est donc à un véritable hôtel « de campagne » que l'on a ici à faire. Les ducs Louis cherchèrent avant tout à pouvoir, à tout moment, se reposer sur des alliés fidèles. Par là même, ils ne pouvaient donc investir ces derniers de charges qui les éloigneraient trop fortement de leur cour.

Agir de la sorte, était-ce, pour autant, une renonciation univoque à toute possibilité immédiate d'obtenir un État centralisé? Une soumission aux usages napolitains? Une défaite de princes «modernes », car élèves des rois de France, incapables de vaincre les reliquats anachroniques de la féodalité que seraient les comtes de leur nouveau royaume? Ne doit-on y voir autre chose? En renforçant le pouvoir militaire local de leurs alliés mais néanmoins vassaux, les ducs d'Anjou prévoyaient certainement de parvenir à structurer un réseau fortifié dont ils pourraient tirer profit à la fois pour assurer leur pouvoir sur le royaume et pour disposer de bases d'attaque, et de repli, pour les campagnes qu'ils auraient à conduire contre leurs rivaux. Adoptant en cela ce fameux « réflexe obsidionnal » qui structure à l'époque la pensée stratégique, ils entendaient confier ces places fortes à des fidèles déjà bien implantés dans un terroir donné, et donc peutêtre plus à même d'y être militairement efficaces que des éléments étrangers qui auraient à y établir leur pouvoir avant d'être en mesure de mener la moindre action. Si la centralisation est ouvertement négligée par les ducs d'Anjou, leurs actions aboutissent à une collaboration efficace $^{73}$, même si elle n'est pas sans anicroche, qui a dû être commandée par une certaine idée du pragmatisme.

Cela étant, il ne faudrait pas négliger le fait que, dès lors que des campagnes avaient à être menées, leurs responsables étaient, le plus souvent, choisis hors des rangs des feudataires napolitains. Ainsi, Louis Ier mène la guerre en personne, Louis II est accompagné de vassaux ou de proches du pape et des conseillers angevins entourent Louis III $^{74}$. Parmi cette élite militaire ou cet «État-major », on peut citer les noms de Raymond d'Agoût, fidèle de Jeanne Ière, honoré de la charge d'amiral de la mer par Louis Ier d'Anjou au moment de son départ en campagne ${ }^{75}$ ou de Juan Gonsalvi, marin espagnol qui fut, entre autres, capitaine général en mer de Louis II $^{76}$. C'est la même chose sur terre où l'on rencontre l'Angevin Jean Chaperon comme maréchal de l'ost de Louis I ${ }^{\mathrm{er} 77}$, le chevalier Louis de Longny maréchal de Louis $\mathrm{II}^{78}$ ou le chambellan-conseiller de Louis III Louis de Beauvau comme lieutenant $a d$ guerram de ce dernier ${ }^{79}$. Il semble donc que les ducs aient jugé préférable de soumettre leurs armées, en ce compris les troupes conduites par les feudataires napolitains, à l'autorité suprême d'un homme de guerre «français » ou provençal dont ils avaient déjà pu apprécier les qualités, ou, et en cela on trouve une réelle adaptation à la pratique de la guerre dans l'Italie médiévale, d'un condottiere, à l'image de Muzzio Attendolo Sforza ou de son fils Francesco, dont les carrières furent intimement mêlées à l'histoire du royaume de Naples. Francesco passa ainsi du service de Jeanne à celui de Louis III et reçut, en échange de ses bandes mercenaires, la fonction de vice-roi de Calabre alors même qu'il entamait le blocus de Naples ${ }^{80}$. Au vrai, une pareille attitude s'était déjà rencontrée dans le cas d'Othon de Brunswick, capitaine d'aventure et quatrième et dernier époux de la reine Jeanne $\mathrm{I}^{\text {ìe }}$ qui, au milieu de ses multiples revirements d'alliance, fut fait en 1386 general capitaine sur le fait de la conqueste pour Madame [Marie de Blois] (...) contre Charle [III de Duras] en Sicile, c'est-à-dire dans le royaume de Naples ${ }^{81}$. À la question du pourquoi d'un tel choix, on pourrait répondre que, les feudataires pouvant être plus sensibles aux approches d'un prétendant au trône avec lequel, dans le cas d'une défaite 
angevine, ils pourraient être amenés à composer, peut-être les ducs ont-ils crus trouver dans les capitaines d'aventure des hommes d'armes qui mettraient plus de temps avant de renoncer à leur serment. Il fallait toutefois, pour cela, bénéficier de ressources économiques que les Angevins échouèrent, à plusieurs reprises, à mobiliser ${ }^{82}$. Il nous semble donc difficilement envisageable de considérer les choses sous cet angle. Notre hypothèse est plutôt que si le souverain acceptait de renforcer son aristocratie féodale en augmentant ses prérogatives via la concession de charges « royales », il refusait d'entièrement se soumettre à elle lorsqu'il s'agissait de conduire la guerre. En tout cela, il y a une volonté de ne pas séparer l'exercice du pouvoir militaire, ou plus exactement la conduite de la guerre, de la personne du souverain, fut-ce même de façon virtuelle lors des années d'enfance de Louis II où le jeune duc est présent aux côtés de ses capitaines qui agissent officiellement comme ses représentants ${ }^{83}$. En plus de s'assurer un relais efficace en campagne, les ducs d'Anjou pouvaient de la sorte écarter le risque des querelles personnelles ou de préséance qui auraient pu éclater entre des comtes et des ducs toujours enclins à agir comme le premier baron du royaume, et ce d'autant plus qu' avec le temps ceux-ci se sont constitués de véritables compagnies d'aventure, comparables à celles des condottieri $^{84}$, ainsi que c'est par exemple le cas d'Onorato Caetani, comte de Fondi, présent aux côtés de Louis ${ }^{\mathrm{er} 85}$. Car même si le service féodal a presque totalement disparu dans le royaume de Naples en faveur de l'adoa, ou adoha - soit le paiement d'une taxe devant permettre de financer les campagnes militaires du souverain ${ }^{86}-$, cela ne signifiait pas que les feudataires ne sortaient jamais les armes. Mais lorsque c'était le cas, c'était avant tout pour défendre leurs intérêts propres, comme l'illustre leur volonté d'obtenir avant tout les charges militaires « situées » sur leurs territoires. C'est pour cela que les Sanseverino suivent les Valois - ils ont intérêt à ce que ce soit cette dynastie qui vainque - et que la place des feudataires est importante dans les guerres civiles. Pour autant, ils ne peuvent certainement pas constituer tout le cadre de l'appareil militaire napolitain, ce qui explique que l'on fasse appel à des condottieri ou à des nobles originaires de l'apanage ou de Provence. Il y a là, manifestement, une volonté des Angevins de jouer sur deux tableaux, à savoir, dans un premier temps, renforcer une aristocratie indispensable à toute conquête de Naples et, dans un second, réserver une part des offices « centraux » (comme celui de Grand Justicier ${ }^{87}$ ou de connétable, chancelier, etc.) à des « Français » ou en tout cas à des non-Napolitains, pour éviter qu'au niveau gouvernemental il ne faille trop souvent composer avec des feudataires dont la puissance, entre autres militaires, pouvait être supérieure à la leur ${ }^{88}$.

On l'aura constaté, une réelle continuité se dessine dans les rapports des ducs d'Anjou avec les offices et les officiers à caractère militaire du royaume de Naples. Cette pérennité d'un système est certes parfaitement éloignée de ce qui, à la même époque, s'organisait au royaume de France, à savoir un appareil militaire centralisé et dépendant directement du pouvoir royal ${ }^{89}$. Toutefois, elle ne peut, nous semble-t-il, être le seul fruit du hasard, ne serait-ce qu'au vu de la part prise par les ducs d'Anjou dans les entreprises militaires et politiques des rois de France Charles V, Charles VI et Charles VII. Tout à fait au courant, donc, des avantages de cette centralisation, auraient-ils pu les ignorer alors même qu'ils avaient eux-mêmes un royaume, certes partiellement en devenir, à gouverner? Il nous semble que le fait d'accepter les exigences des habitants du royaume de Naples plutôt que de les contraindre à adopter des cadres politiques qui leur étaient tout à fait étrangers relève moins d'une incapacité à soumettre ces feudataires que d'une compréhension des réalités socio-politiques du royaume de Naples et, de ce fait, d'un choix orienté par un certain pragmatisme. Si Louis Ier n'avait aucune connaissance du royaume de Naples au moment de franchir les Alpes, le fait que ses successeurs l'imitent dans leurs décisions nous incite à penser que se développa, à la cour d'Anjou, un programme de gouvernement du royaume de Naples, programme qui se diffusa de père en fils via des canaux que nous allons maintenant tenter d'identifier.

Dans un premier temps, il paraît envisageable que l'un des ducs d'Anjou de la maison Valois ait, à un moment de ses tentatives napolitaines, fait réaliser un ouvrage comparable au Memoire pour faire l'instruction de ceux qui vont à Jennes composé à l'intention de ceux que le roi de France envoyait en Ligurie au début du XVe siècle ${ }^{90}$. Cet ouvrage, en livrant, sous la forme 
d'un récit assez détaillé, les accidents avec lesquels le pouvoir français s'était établi sur la République, devait permettre à ses lecteurs de maitriser les tenants et les aboutissants des tensions qui structuraient la vie politique génoise. Dans le cas des ducs d'Anjou, toutefois, on ne trouve pas, du moins d'après ce que laissent connaître des sources éparses où ne figurent pas de catalogue de leur librairie, d'ouvrage de ce genre ${ }^{91}$. Toutefois, ces silences doivent plutôt nous inciter « à considérer le caractère très partiel de nos sources qu'à remettre en cause [...] la richesse de [leur] librairie ${ }^{92}$. » À moins que ce livre dont nous supposons l'existence n'ait été abandonné à la fin d'une campagne malheureuse, comme celle de Louis III en 1434 ou de René huit ans plus tard...

Une autre possibilité pouvant expliquer la répétition des formules que nous venons de mettre en avant - elle est d'ailleurs tout à fait conciliable avec la première - serait de voir dans les duchesses d'Anjou les relais de cette politique particulière. Alors que son mari guerroyait dans le royaume de Naples, Marie de Blois gouverna ses possessions cisalpines et agit comme une parfaite régente, et ce surtout en Provence où elle s'opposa à l'Union d'Aix ${ }^{93}$. Il n'est donc pas à exclure que, informée régulièrement des avancées de son époux par le biais de coursiers dont certains faisaient halte à Avignon ${ }^{94}$, elle ait également reçu nombre d'informations sur le royaume de Naples. Soit des informations qu'elle aurait pu transmettre à son fils Louis avant que celui-ci ne prenne à son tour la route de l'Italie. Le jeune âge de ce dernier au moment de sa première campagne - il a moins de 13 ans en 1390 et son père, mort alors qu'il n'avait que sept ans, n'eut guère le temps de lui enseigner les subtilités du gouvernement napolitain - rend probable la diffusion de ce savoir à d'autres membres de son entourage, sans négliger le fait que le poids des feudataires et conseillers régnicoles fut, au cours des dix années qui le séparèrent de son retour en France, pour le moins prépondérant dans sa politique. Notons toutefois qu'après 1409 , à un moment où il était en mesure de conduire une politique personnelle, il adopta à nouveaux ces «techniques », preuve qu'il devait être convaincu de leur efficacité. Au retour de sa première expédition, il put partager ces connaissances avec celle qui devint son épouse en 1400, Yolande d'Aragon. Yolande ou Louis, décédé en 1417, ne manquèrent certainement pas de former leur fils et héritier, Louis III, né en 1403, à gouverner ce royaume de Naples qui tenait un rôle capital dans leurs existences ${ }^{95}$. Selon toute vraisemblance, mais cela dut plutôt être le fait de la duchesse, il en fut de même avec leur puîné René, né en 1409. En conclusion, le gouvernement militaire des ducs d'Anjou sur le royaume de Naples se signale par trois traits communs tout au long du demi-siècle d'histoire que nous avons étudié. Tout d'abord, les mécanismes de ce pouvoir demeurent semblables au fil du temps, preuve d'un ralliement clair, bien que peut-être en partie sous la contrainte, à une manière de faire la guerre au royaume de Naples. Ensuite, les offices militaires à vocation géographique limitée (châtelain, capitaine, portulan) sont le plus souvent confiés à des régnicoles qui avaient des intérêts dans les régions où ils exerçaient le pouvoir délégué par le roi de Naples (fiefs ou autres possessions foncières, rentes...). L'État napolitain s'organise donc, et ici la dimension militaire peut faire écho à d'autres réalités, autour d'un processus de négociation entre celui qui est censé le diriger, le roi, et ceux qui sont censés lui obéir, ses vassaux, selon une dialectique qui semble d'ailleurs commune à la Méditerranée médiévale ${ }^{96}$. Enfin, les officiers en charge de la conduite des armées en campagne, ou des places intimement liées au souverain (châteaux ou province de résidence...), sont au contraire, et sauf exception, des étrangers au royaume de Naples, qu'ils proviennent de l'apanage, de Provence ou d'autres régions de la péninsule italienne. Ces deux derniers éléments soulignent donc la part capitale prise par les hommes dans l'évolution de l'« État napolitain » et de ses structures. C'est en effet le rapport de force entre prince et féodaux qui a forgé les fondements administratifs du royaume de Naples. Ceux-ci ne sont donc pas perturbés par l'action des princes Valois. Seul le processus de monopolisation royale du pouvoir de conduire la guerre, qui se développe au même moment en France ${ }^{97}$, témoigne d'une mainmise angevine. 


\section{Bibliographie}

Albanès 1875 = J.-H. Albanès, La bibliothèque du roi René, dans Revue des sociétés savantes des départements, $5^{\mathrm{e}}$ sér., 8.2, 1875, p. 301-311.

Arlot $2006=\mathrm{F}$. Arlot, Dans la tourmente du XIV siècle. Marie de Blois, comtesse de Provence et reine de Naples, dans Provence historique, 56, 2006, p. 53-89, 155-194.

Arnone $1893=\mathrm{N}$. Arnone, Le regie tombe del duomo di Cosenza, dans Archivio storico per le Province Napoletane, 18, 1893, p. 380-408.

Avril 2009 = F. Avril, L'héritage : quelques livres des premiers ducs d'Anjou, dans M.-É. Gautier (dir.), Splendeur de l'enluminure. Le roi René et ses livres, Angers-Arles, 2009, p. 36-44.

Barone $1887=\mathrm{N}$. Barone, Notizie storiche tratte dai Registri di Cancellaria di Carlo III di Durazzo, dans Archivio storico per le province napoletane, 12, 1887, p. 5-30, 184-208.

Beltrani 1956 = G. Beltrani, Gli Orsini di Lecce e di Taranto durante il regno di Giovanna II, dans Archivio storico per le province napoletane, 36, 1956, p. 93-125.

Bénet 1997 = J. Bénet, Jean d'Anjou, duc de Calabre et de Lorraine (1426-1470), Nancy, 1997.

Bidet $2000=\mathrm{L}$. Bidet, La noblesse et les princes d'Anjou. La famille de Beauvau, dans N. Coulet, J.-M. Matz (dir.), La noblesse dans les territoires angevins à la fin du Moyen Âge. Actes du Colloque international organisé par l'Université d'Angers. Angers-Saumur, 3-6 juin 1998, Rome, 2000, p. 471-497.

Bisson de Sainte-Marie 1884 = R. Bisson de Sainte-Marie, Testament de Jacques de Tarente, dernier empereur de Constantinople, en faveur de Louis d'Anjou (15 juillet 1383), dans Bibliothèque de l'École des chartes, 45, 1884, p. 189-195.

Borghese 2011 = G. L. Borghese, L'ombre portée d'archives disparues. Travailler sur le Royaume de Naples après la destruction des Registres angevins (30 septembre 1943), dans Cahiers électroniques d'histoire textuelle du LAMOP, 4, L'absence de texte, 2011, p. 20-39.

Bresc, Veauvy 1990 = H. Bresc, C. Veauvy, Genèse de l'État moderne en Méditerranée, dans J.-P. Genet (dir.), L'État moderne : Genèse. Bilans et perspectives. Actes du Colloque tenu au CNRS à Paris les 19-20 septembre 1989, Paris, 1990, p. 75-92.

Caridi 1995 = G. Caridi, La spada, la seta, la croce. I Ruffo di Calabria dal XIII al XIX secolo, Turin, 1995.

Caridi 1999 = G. Caridi, I Ruffo di Calabria (secoli XIII - XIX), Reggio Calabria, 1999.

Celani 1895 = E. Celani, Documenti sforzeschi nell'Archivio di Stato di Napoli, dans Archivio storico Lombardo, $3^{\text {e }}$ sér., 4, 1895, p. 377-386.

Chaigne-Legouy 2013 = M. Chaigne-Legouy, «Pays de par-deçà, pays de par-delà ». Les relations entre Angevins et Napolitains sous le regard de Jean Le Fèvre, chancelier de la seconde maison d'Anjou (1380-1388), dans A. Lemonde, I. Taddei (dir.), Circulation des idées et des pratiques politiques. France et Italie (XIII'-XVI siècle), Rome, 2013, p. 149-186.

Chronicon Siculum 1887 = Chronicon Siculum incerti authoris ab anno 1340 ad annum 1396, éd. J. De Blasiis, Naples, 1887.

Colapietra 1997 = R. Colapietra, Ombre e luci nell'autunno del Medio Evo : il regno di Napoli nel periodo durazzesco, dans Rassegna storica Salernitana, 27, 1997, p. 43-68.

Contamine 1972 = P. Contamine, Guerre, État et société à la fin du Moyen Âge. Études sur les armées des rois de France 1337-1494, Paris, 1972.

Cortez 1921 = F. Cortez, Les grands officiers royaux de Provence au Moyen Âge, Aix-en-Provence, 1921.

Coulet $2005=$ N. Coulet, 1380-1482. L'ultime principauté de Provence ou la seconde maison d'Anjou, dans M. Aurell, J.-P. Boyer, N. Coulet, La Provence au Moyen Âge, Aix-en-Provence, 2005, p. 281-328.

Coville 1941 = A. Coville, La vie intellectuelle dans les domaines d'Anjou-Provence de 1380 à 1435 , Paris, 1941.

Cozzetto 1987 = F. Cozzetto, Territorio, istituzioni e società nella Calabria moderna, Naples, 1987.

Cruciani 1986 = M. G. Cruciani, Calabria Citeriore, dans G. Galasso, R. Romeo (dir.), Storia del Mezzogiorno, VII, Le Province, Rome, 1986, p. 241-301.

Cutolo 1977 = A. Cutolo, Maria d'Enghien, regina di Napoli, 2e éd., Galatina, 1977.

De Boüard 1936 = M. de Boüard, Les origines des Guerres d'Italie. La France et l'Italie au temps du Grand Schisme d'Occident, Paris, 1936. 
Dietrich de Nieheim 1890 = Dietrich de Nieheim, De scismate libri tres, éd. G. Erler, Leipzig, 1890.

I diurnali del duca di Monteleone 1960 = I diurnali del duca di Monteleone, éd. M. Manfredi, dans Rerum italicarum scriptores. Raccolta degli storici italiani dal cinquecento al millecinquecento ordinata da L. A. Muratori. Nuova edizione riveduta ampliata e corretta, XXI.V, Bologne, 1960.

Doüet d'Arcq 1863 = L. Doüet d'Arcq, Choix de pièces inédites relatives au règne de Charles VI, I, Paris, 1863.

Durrieu 1885 = P. Durrieu, Les Gascons en Italie. Études historiques, Auch, 1885.

Faraglia 1894 = N. F. Faraglia, Studii intorno al regno di Giovanna II, dans Atti della Accademia Pontaniana, 24.16, 1894.

Favier 2008 = J. Favier, Le roi René, Paris, 2008.

Feliciangeli 1907 = B. Feliciangeli, Sul passagio di Luigi I d'Angio e di Amedeo VI di Savoia attraverso la Marca e l'Umbria (1382), dans Atti e memorie della R. deputazione di Storia patria per le Marche, 4, 1907, p. 369-462.

Galasso 1992 = G. Galasso, Storia d'Italia, XV.I, Il Regno di Napoli. Il Mezzogiorno angioino e aragonese (1266-1494), Turin, 1992.

Gautier 2009 = M.-É. Gautier, La bibliothèque du roi René, dans Id. (dir.), Splendeur de l'enluminure. Le roi René et ses livres, Angers-Arles, 2009, p. 20-35.

Giovanni Antonio Campano 1929 = Giovanni Antonio Campano, Bracii Perusini Vita et Gesta ab anno MCCCLXVIII usque ad MCCCCXXIV, éd. R. Valentini, dans Rerum italicarum scriptores. Raccolta degli storici italiani dal cinquecento al millecinquecento ordinata da L. A. Muratori. Nuova edizione riveduta ampliata e corretta, XIX.IV, Bologne, 1929.

Istore et croniques de Flandres $1880=$ Istore et croniques de Flandres, d'après les textes de divers manuscrits, II, éd. J. B. M. C. Kervyn de Lettenhove, Bruxelles, 1880.

Jacopo Salviati 1784 = Jacopo Salviati, Cronica, o memorie dall'Anno 1398. al 1411, éd. I. Di San Luigi, Delizie degli eruditi toscani, 18, 1784, p. 175-361.

Jarry 1896 = E. Jarry, Les origines de la domination française à Gênes (1392-1402), Paris, 1896.

Jean Froissart 1869 = Jean Froissart, Chroniques, IX, 1377-1382, éd. J. B. M. C. Kervyn de Lettenhove, Bruxelles, 1869.

Jean Le Fèvre 1887 = Jean Le Fèvre, Journal, I, éd. H. Moranvillé, Paris, 1887.

Kiesewetter 2001 = A. Kiesewetter, Ricerche e documenti per la signoria di Raimondo del Balzo-Orsini sulla contea di Lecce e sul principato di Taranto (1385-1399/1406), dans Bollettino storico di Terra d'Otranto, 11, 2001, p. 17-30.

Kiesewetter 2005 = A. Kiesewetter, Problemi della signoria di Raimondo del Balzo Orsini in Puglia (1386-1406), dans G. Carducci, A. Kiesewetter, G. Vallone, Studi sul Principato di Taranto in età orsiniana, Bari, 2005, p. 7-88.

Labande 1932 = E.-R. Labande, Le rôle de Rinaldo Orsini dans la lutte entre les papes de Rome et d'Avignon (1378-1390), dans Mélanges d'Archéologie et d'Histoire. École française de Rome, 49, 1932, p. $157-180$.

Labande 1939 = E.-R. Labande, Rinaldo Orsini, comte de Tagliacozzo $(\dagger 1390)$ et les premières guerres suscitées en Italie centrale par le Grand Schisme, Monaco-Paris, 1939.

Labande 1951 = E.-R. Labande, Une oraison funèbre inconnue de Louis I ${ }^{\mathrm{er}}$ d'Anjou, roi de Sicile, dans Bibliothèque de l'École des chartes, 109, 1951, p. 42-50.

Lecoy de La Marche 1873 = A. Lecoy de La Marche (dir.), Extraits des comptes et mémoriaux du roi René pour servir à l'histoire des arts au XV $V^{\mathrm{e}}$ siècle publiés d'après les originaux des Archives nationales, Paris, 1873.

Lefèvre 2006 = S. Lefèvre, Antoine de La Sale. La fabrique de l'œuvre et de l'écrivain. Suivi de l'édition critique $d u$ Traité des anciens et des nouveaux tournois, Genève, 2006.

Leodrisius Crivelli 1731 = Leodrisius Crivelli, De vita rebusque gestis Sfortiae belicosissimi ducis, ac initiis filii ejus Francisci Sfortiae Vicecomitis, Mediolanensium ducis, commentarius ab anno circiter MCCCLXIX usque ad MCCCCXXIV, dans L. A. Muratori, Rerum italicarum scriptores ab anno aerae christianae quingentesimo ad millesimumquingentesimum, quorum potissima pars nunc primum in lucem prodit ex ambrosianae, estensis, aliarumque insignium bibliothecarum codicibus, XIX, Milan, Societas Palatina, 1731, col. 627-732. 
Léonard 1924 = é.-G. Léonard, La captivité et la mort de Jeanne $I^{\text {re }}$ de Naples, dans École française de Rome, Mélanges d'Archéologie et d'Histoire, 41, 1924, p. 43-77.

Lorenzo Bonincontri 1732 = Lorenzo Bonincontri, Miniatensis Annales ab anno MCCCLX usque ad MCCCCLVIII, dans L. A. Muratori, Rerum italicarum scriptores ab anno aerae christianae quingentesimo ad millesimumquingentesimum, quorum potissima pars nunc primum in lucem prodit ex ambrosianae, estensis, aliarumque insignium bibliothecarum codicibus, XXI, Milan, Societas Palatina, 1732, col. 99-162.

Mancarella 1920 = A. Mancarella, Firenze, la Chiesa e l'avvenato di Ladislao di Durazzo al trono di Napoli, dans Archivio storico per le province napoletane, 45, 1920, p. 28-60.

Mafrici 1986 = M. V. Mafrici, Calabria Ulteriore (1266-1860), dans G. Galasso, R. Romeo (dir.), Storia del Mezzogiorno, VII, Le Province, Rome, 1986, p. 95-232.

Massaro 2005 = C. Massaro, Città e territorio nella contea di Lecce, dans G. Vitolo (dir.), Città e contado nel Mezzogiorno tra Medioevo ed Età moderna, Salerno, 2005, p. 229-247.

Masson 2014 = C. Masson, Des guerres en Italie avant les Guerres d'Italie. Les entreprises militaires françaises dans la Péninsule à l'époque du Grand Schisme d'Occident, Rome, 2014.

Mazzoleni 1938 = J. Mazzoleni, Le pergamene dell'Archivio della R. Camera della Sommaria e la loro importanza per la storia delle Puglie (1267-1458), dans Japigia, $\mathrm{n}^{\text {le }}$ sér., 9, 1938, p. 281-306.

Miceli di Serradileo 1976 = A. Miceli di Serradileo, Una dichiarazione di Luigi III d'Angiò dalla città di San Marco in Calabria, dans Archivio storico per la Calabria e la Lucania, 43, 1976, p. 69-81.

Monteleone 2010 = F. Monteleone, Maria d'Enghien, contessa di Lecce. Dimensione umana e vicenda politica, dans P. Mainoni (dir.), "Con animo virile ». Donne e potere nel Mezzogiorno medievale (secoli XI-XV), Rome, 2010, p. 319-359.

Moscati 1936 = R. Moscati, Ricerche e documenti sulla feudalità napoletana nel periodo angioino, dans Archivio storico per le province napoletane, 61, 1936, p. 1-14.

Orefice 1982 = I. Orefice, I Registri della cancellaria angioina ricostruiti da Riccardo Filangieri con la collaborazione degli archivisti napoletani, XXXIV, 1431-1434, Naples, 1982.

Pacella 1964 = F. Pacella, Un barone condottiero della Calabria del sec. XIV-XV : Nicolò Ruffo, marchese di Cotrone, conte di Catanzaro, dans Archivio storico per le province napoletane, 82, 1964, p. 45-93.

Pellettieri-Legouy 1991 = A. Pellettieri-Legouy, Ugo Sanseverino, un feudatario della Basilicata nella lotta tra Angioini e Durazzeschi, dans Rassegna storica Lucana, 14, 1991, p. 61-78.

Perrat 1945 = C. Perrat, Les archives d'État de Naples et l'histoire de France, dans Comptes rendus des séances de l'Académie des Inscriptions et Belles-Lettres, 89, 1945, p. 321-333.

Pollastri 1991 = S. Pollastri, Une famille de l'aristocratie napolitaine sous les souverains angevins : les Sanseverino (1270-1420), dans MEFRM, 103-1, 1991, p. 237-260.

Pollastri 2005 = S. Pollastri, Enquête sur les droits de justice de l'aristocratie napolitaine (XIV $X V^{\mathrm{e}}$ siècles). Quelques exemples, dans J.-P. Boyer, A. Mailloux, L. Verdon (dir.), La justice temporelle dans les territoires angevins aux XIII ${ }^{\mathfrak{e}}$ et XIV ${ }^{\mathfrak{e}}$ siècles. Théories et pratiques, Rome, 2005, p. 279-305.

Pollastri $2006=\mathrm{S}$. Pollastri, Les relations entre feudataires et villes du littoral en Calabre au XIV siècle, dans P. Jansen (dir.), Entre monts et rivages. Les contacts entre la Provence orientale et les régions voisines au Moyen Age, Antibes, 2006, p. 249-264.

Pollastri 2013 = S. Pollastri, L'aristocratie comtale sous les Angevins (1265-1435), dans MEFRM [en ligne], 125.1, 2013 [URL : http://mefrm.revues.org/1110].

Pontieri 1958 = E. Pontieri, Muzio Attendolo e Francesco Sforza nei conflitti dinastico-civili nel regno di Napoli al tempo di Giovanna II d'Angiò-Durazzo, dans Studi storici in onore di Gioacchino Volpe per il suo $80^{\circ}$ compleano, II, Florence, 1958, p. 787-883 [rééd. dans Divagazioni storiche e storiografiche, I, Naples, 1960, p. 73-199].

Pontieri 1961 = E. Pontieri, Fonti Aragonesi, II, I registri della Cancellaria Vicereale di Calabria (1422-1453), Naples, 1961.

Pontieri 1963 = E. Pontieri, La Calabria a metà del secolo XV e le rivolte di Antonio Centelles, Naples, 1963.

Pontieri 1982 = E. Pontieri, Introduzione, dans I. Orefice, I Registri della cancellaria angioina ricostruiti da Riccardo Filangieri con la collaborazione degli archvisiti napoletani, XXXIV, 1431-1434, Naples, 1982, p. IX-XL. 
Reynaud 2000 a = M.-R. Reynaud, Le temps des princes. Louis II \& Louis III d'Anjou-Provence, 1384-1434, Lyon, 2000.

Reynaud $2000 \mathrm{~b}=$ M.-R. Reynaud, Noblesse et pouvoir dans la principauté d'Anjou-Provence sous Louis II et Louis III (1384-1434), dans N. Coulet, J.-M. Matz (dir.), La noblesse dans les territoires angevins à la fin du Moyen Âge. Actes du Colloque international organisé par l'Université d'Angers. Angers-Saumur, 3-6 juin 1998, Rome, 2000, p. 303-313.

Schnerb 2005 = B. Schnerb, Jean sans Peur. Le prince meurtrier, Paris, 2005.

Selzer 2001 = S. Selzer, Deutsche Söldner im Italien des Trecento, Tübingen, 2001.

Sozomenus 1730 = Sozomenus, Specimen historiae ab anno Christi MCCCLXII usque ad MCCCCX, dans L. A. Muratori, Rerum italicarum scriptores ab anno aerae christianae quingentesimo ad millesimumquingentesimum, quorum potissima pars nunc primum in lucem prodit ex ambrosianae, estensis, aliarumque insignium bibliothecarum codicibus, XVI, Milan, Societas Palatina, 1730, col. 1063-1198.

Trifone 1962 = R. Trifone, Gli organi dell'amministrazione angioina, dans Archivio storico pugliese, 15,1962 , p. 83-100.

Uginet 1980 = F. C. Uginet, Challant, Boniface de, dans Dizionario biografico degli Italiani, XXIV, 1980, p. 361-362.

Valois 1902 = N. Valois, La France et le Grand Schisme d'Occident, IV, Paris, 1902

Vitale 2003 = G. Vitale, Élite burocratica e famiglia. Dinamiche nobiliari e processi di costruzione statale nella Napoli angioino-aragonese, Naples, 2003.

\section{Notes}

1 Sur ces campagnes angevines, voir, entre autres, Galasso 1992, p. 234-237, 249-255, 268-272, 293-307, 561-587, 642-663 ; Masson 2014 (pour Louis I ${ }^{\text {er }}$ et Louis II) ; Pontieri 1963, p. 12-20, 120-121 et Id. 1982 (pour Louis III) ; Favier 2008, p. 54-55, 78-103 (pour René); Benet 1997 (pour Jean d'Anjou).

2 Il serait intéressant de consacrer une autre étude de ce type à la période des tentatives angevines pour jeter les Aragonais à bas d'un trône dont ces derniers étaient parvenus à s'emparer après la disparition de la reine Jeanne II.

3 Borghese 2011, p. 22-23 ; Perrat 1945, p. 323-324.

4 On signalera ainsi le rôle capital joué dans cette recherche par les collections de l'Archivio segreto Vaticano (=ASV) qui conserve entre autres la comptabilité des papes d'Avignon, extrêmement actifs dans le financement des premières campagnes angevines.

5 Des familles telles que les Craon ou les Beauvau, pour le premier groupe, les Agoût, pour le second, enverront certains de leurs plus batailleurs enfants accompagner leur prince outremonts.

6 Parmi les sujets napolitains, on peut retenir l'exemple de la famille del Giudice, appelée « Juge » par les écrivains et les scribes français comme avignonnais, dont l'un des membres, Jacomino, apparaît comme capitaine d'arbalétriers dans l'armée de Louis I ${ }^{\text {er }}$. ASV, Camera apostolica (=Cam. Ap.), Introitus et Exitus (=Intr. et Ex.) 337, fo $52 \mathrm{v}^{\circ}$. Contamine 1972, p. 558 l'a repéré dans les armées royales françaises de 1379 à 1388, ce qui explique, à tout le moins en partie, son ralliement aux prétentions angevines.

7 Braccio da Montone fut ainsi présent aux côtés de Louis II lors de ses dernières campagnes des années 1409-1411. Florence, Archivio di Stato (=ASFi), Consulte e pratiche, registro 40, $\mathrm{f}^{\circ} 87 \mathrm{v}^{\circ}$; ASFi, Signori. Legazione e Commissarie, Elezioni, Istruzioni, Lettere, $\mathrm{n}^{\circ} 4, \mathrm{f}^{\mathrm{o}} 110 \mathrm{r}^{\circ}-\mathrm{v}^{\mathrm{o}}, 111 \mathrm{v}^{\mathrm{o}}-113 \mathrm{r}^{\mathrm{o}}$; Sienne, Archivio di Stato (=ASSi), Concistoro. Carteggio 270, $\mathrm{f}^{\circ} 58 \mathrm{r}^{\circ}$; I diurnali del duca di Monteleone 1960, p. 83 ; Giovanni Antonio Campano 1929, p. 44-45, 50-53 ; Jacopo Salviati 1784, p. 344-348 ; Lorenzo Bonincontri 1732, col. 103 ; de Boüard 1936, p. 373-374 ; Valois 1902, p. 123, 134, 138-140.

8 Retenons ici le nom de Guillaume de Fellimbach, qui suivit Louis d'Anjou tout en demeurant membre de la compagnie de Saint-Georges d'Alberigo da Barbiano, condottiere au service des Duras, ce qui n'est pas sans éclairer la pratique de la guerre de ces troupes d'aventures. Dietrich de Nieheim 1890, p. 53 ; Jean Le Fèvre 1887, p. 279 ; Feliciangeli 1907, p. 454 ; Selzer 2001, p. 325.

9 Alain de Saint-Pol est ainsi aux ordres, en tant que capitaneus certarum gentium armorum de Societate Britonum, de Louis $\mathrm{I}^{\mathrm{er}}$ tandis que, en 1387, les Pouilles voient opérer, officiellement pour le service de Louis II, la compagnie de Bertrand Guillaume. ASV, Instrumentum miscellaneum (=Instr. misc.) 3242, $\mathrm{n}^{\circ} 19,22$.

10 Jean Froissart 1869, p. 469 ; Jean Le Fèvre 1887, p. 24 ; Reynaud 2000 b, p. 308-309.

11 Y siégeaient également de nombreux régnicoles. Paris, Archives nationales, P $1334^{17}, \mathrm{n}^{\circ} 35$; Labande 1939, p. 175. 
12 ASSi, Concistoro. Carteggio 1843, n 93 ; Sozomenus 1730, col. 1166 ; de Boüard 1936, p. 236 ; Durrieu 1885, p. 206.

13 Caridi 1999, p. 48 indique qu'il ne conserve guère longtemps ce titre. Folco Ruffo, qui en avait été déchu par Louis $\mathrm{I}^{\mathrm{er}}$ à la suite de son soutien à Charles de Duras, en reprit en effet rapidement possession. 14 Le 10 mai 1382. Jean Le Fèvre 1887, p. 35 ; Chaigne-Legouy 2013, p. 161.

15 ASV, Cam. Ap., Intr. et Ex. 337, fo $96 \mathrm{v}^{\circ}$; ASV, Cam. Ap., Intr. et Ex. 359, fo $154 \mathrm{r}^{\circ}$; Paris, Bibliothèque nationale de France, Manuscrit français 20384, $\mathrm{n}^{\circ} 28$; Chronicon Siculum 1887, p. 97, 110-111, 115-116 ; I diurnali del duca di Monteleone 1960, p. 33 ; Istore et croniques de Flandres 1880, p. 360 ; Lorenzo Bonincontri 1732, col. 42 ; Barone 1887, p. 28 ; Lefèvre 2006, p. 238, 240.

16 La Calabre était à l'époque dominée par la puissance de ce lignage. Pacella 1964, p. 56.

17 Kiesewetter 2001, p. 22.

18 Miceli di Serradileo 1976, p. 80 n. 25.

19 ASV, Cam. Ap., Intr. et Ex. 366, fo 55 r $^{\circ}$; ASV, Cam. Ap., Intr. et Ex. 370, fo 84 r $^{\circ}$; ASV, Instr. misc. 3337, $\mathrm{n}^{\circ} 64$; ASV, Registra Avignonesi (=Reg. Aven.) 254, f $266 \mathrm{r}^{\circ}-\mathrm{v}^{\mathrm{o}}, 327 \mathrm{v}^{\circ}$; ASV, Reg. Aven. 335, f $735 \mathrm{r}^{\circ}$; Léonard 1924, p. 70.

20 Jean Le Fèvre 1887, p. 122 ; Cortez 1921, p. 82.

21 Par Louis I $^{\text {er }}$ d'Anjou. Labande 1951, p. 47-48.

22 Bisson de Sainte-Marie 1884.

23 ASV, Cam. Ap., Intr. et Ex. 362, f 82 v $^{\circ}$ ASV, Instr. misc. 5774 ; ASV, Reg. Aven. 254, fo 259 r $^{\circ}$, $323 \mathrm{v}^{0}$; I diurnali del duca di Monteleone 1960, p. 46 ; Jean Le Fèvre 1887, passim ; Chaigne-Legouy 2013, p. 162, 165-173, 176, 178-183, 186 ; Pellettieri-Legouy 1991, p. 65.

24 À ce sujet, voir notamment Pontieri 1958, p. 836.

25 Gerace, prov. Reggio Calabria, rég. Calabre, Italie. Mazzoleni 1938, p. 300 ; Orefice 1982, p. 83.

26 C'est à ce titre qu'il avait rejoint l'armée de Louis $\mathrm{I}^{\text {er }}$ d'Anjou.

27 Chronicon Siculum 1887, p. 95 ; I diurnali del duca di Monteleone 1960, p. 57 ; Mancarella 1920, p. 58.

28 Chaigne-Legouy 2013, p. 160.

29 Pollastri 2013, § 97.

30 Chaigne-Legouy 2013, p. 162.

31 Celani 1895, p. 380.

32 Pollastri 2013, § 93.

33 Largement répandue dans le chef des Angevins, cette habitude de conférer des fiefs ou des offices à conquérir, car encore possédés par des fidèles des Duras, était une façon de maintenir l'enthousiasme de leurs alliés à faire la guerre à ces derniers.

34 Probablement Rigniano Gargano, prov. Foggia, rég. Pouilles, Italie. I diurnali del duca di Monteleone 1960, p. 33 ; Jean Le Fèvre 1887, p. 425.

35 Ibid., p. 227-229 ; Cutolo 1977, p. 28 ; Labande 1939, p. 135.

36 Jean Le Fèvre 1887, p. 282.

37 On conserve en effet son registre où sont consignés les actes qu'il édicta tout au long de son séjour en Italie. Orefice 1982.

38 Arena, prov. Vibo Valentia; Stilo, prov. Reggio Calabria, rég. Calabre, Italie. Orefice 1982, p. 63, 74.

39 Ibid., p. 14.

40 Ibid., p. 88, 92.

41 L'on peut ainsi évoquer, avec Pollastri 2006, p. 249 n. 1 ; Id. 2013, § 83, la figure de plusieurs feudataires se faisant investir de capitaineries situées aux frontières de leurs possessions.

42 Id. 2005, p. 293.

43 Tagliacozzo, prov. L'Aquila, rég. Abruzzo, Italie. Chronicon Siculum 1887, p. 47 ; Labande 1932, p. 169.

44 Bova, prov. Reggio Calabria, rég. Calabre, Italie. Orefice 1982, p. 38 ; Pontieri 1961, p. 17.

45 Caridi 1995, p. 42.

46 Le processus, apparu à l'époque de Ladislas de Duras, connut une phase d'accélération dans les années 1420-1425, qui sont justement celles de l'arrivée de Louis III dans le royaume (Pollastri 2013, $\S 83)$. On constate donc de sa part une capacité à très rapidement s'adapter aux usages locaux.

47 Le 26 juillet 1426. Orefice 1982, p. 76. 
48 Probablement la localité aujourd'hui appelée Aiello Calabro, prov. Cosenza, rég. Calabre, Italie. Orefice 1982, p. 5, 62.

49 Capo Stilo semble avoir disparu aujourd'hui ; Capo Vaticano, comm. Ricadi, prov. Vibo Valentia, rég. Calabre, Italie. Orefice 1982, p. 91.

50 En 1433. Ibid., p. 95.

51 Cette « chasse » eut évidemment aussi lieu dans tous les autres champs d'exercice du pouvoir royal. Pour les droits de justice, voir par exemple Pollastri 2013, § 86-87.

52 Sur ces deux offices, voir, entre autres, Trifone 1962, p. 92.

53 Pollastri 2006, p. 254. Le capitaine était initialement un officier « civil » chargé d'exercer la justice dans les universitates du royaume. Toutefois, au lendemain des Vêpres, et avec une vigueur accrue au moment des conflits entre Angevins et Aragonais, il revêtira, sans perdre ses prérogatives judiciaires, un rôle de plus en plus militaire, marqué entre autres par la responsabilité de la collecte des subventions royales nécessaires à la mise sur pied et au paiement de l'armée, ce en quoi il prend la place des collecteurs royaux. Massaro 2005, p. 234 ; Pollastri 1991, p. 250-251; Id. 2005, p. 282.

54 Orefice 1982, p. 102.

55 Monteleone 2010, p. 333 n. 71.

56 Kiesewetter 2005, p. 18.

57 Vitale 2003, p. 72. Cette auteure utilise l'expression de «élite di potere » pour désigner les feudataires impliqués dans cette collection d'offices.

58 Montesarchio, prov. Benevento, rég. Campanie, Italie. ASV, Cam. Ap., Intr. et Ex. 355, fo 88 r $^{\circ}$; Uginet 1980, p. 362.

59 Nous ne sommes pas parvenu à identifier cette localité. Orefice 1982, p. 46.

60 Ibid., p. 90.

61 S'il faut en croire l'exemple de Robin de Jennes, lieutenant de Tristan de la Jaille. Ibid., p. 90.

62 En 1391-1392. Lefèvre 2006, p. 241.

63 Au titre de capitaine, selon Bidet 2000, p. 473, ou de châtelain, selon Kiesewetter 2005, p. 47 pour qui le capitaine est le Capouan Giordano Pandone. Au vrai, même sous la plume de Jean Le Fèvre 1887, p. 146, 214, 294-295, 383, 476, auteur pourtant bien informé, les deux termes semblent interchangeables dans le cas de Jean de Beauvau, prédécesseur à cette charge de son neveu Macé.

64 Orefice 1982, p. 106, 112, 114, 118, 126.

65 Ibid., p. 72-75.

66 Pontieri 1982, p. XXV, XXXIV.

67 Galasso 1992, p. 302.

68 Colapietra 1997, p. 59 ; Cozzetto 1987, p. 74, 76, 90.

69 Cruciani 1986, p. 247 ; Mafrici 1986, p. 98 ; Pontieri 1963, p. 18 ; Id. 1982, p. XIX.

70 Voir par exemple Coulet 2005, p. 319-321 ; Reynaud 2000 b, p. 24, 166, 170-172, 180, 183.

71 Schnerb 2005, p. 70-74.

72 En 1433-1434. Orefice 1982, p. 113, 121

73 Dans un cadre plus large, voir Pollastri 2013, § 101.

74 On retrouve ce choix lors de la campagne de Jean d'Anjou, fils de René, en 1459-1463, à l'occasion de laquelle il convie le condottiere Jacopo Piccinino à le rejoindre et à compléter son armée de ses troupes (Bénet 1997, p. 80). Celui-ci est pour Jean le capitaine qui pourra conduire la guerre et faire preuve d'une certaine fidélité, nourrie tant par les soldes qui lui sont versées que par l'espoir de se tailler un fief dans le royaume.

75 ASV, Cam. Ap., Intr. et Ex. 355, f 105 vº ; Jean Le Fèvre 1887, p. 26 ; Labande 1939, p. 175.

76 Marseille/Aix-en-Provence, Archives départementales des Bouches-du-Rhône (=ADBdR), B 592, 10 et 26 décembre 1394 ; ADBdR, B 596, 22 juin 1398.

77 ASV, Cam. Ap., Intr. et Ex. 337, f 30 r $^{\circ}$; Doüet d'Arcq 1863, p. 125.

78 ASV, Reg. Aven. 328, f $484 v^{\circ}$.

79 Orefice 1982 , p. 72.

80 Galasso 1992, p. 294. Pour les années 1421 à 1423, le titre de vice-roi lui est attribué par Leodrisius Crivelli 1731, col. 708 et I diurnali del duca di Monteleone 1960, p. 108 et à leur suite par Arnone 1893, p. 398 ; Beltrani 1956, p. 115 ; Pontieri 1963, p. 15-16, 121, 129 alors que ASV, Registri Vaticani 353, $\mathrm{f}^{0}$ CCXXXII $\mathrm{r}^{0}$ ne le qualifie que de nonnullarum gentium armigerum capitaneus ac noster et Romanae Ecclesiae confalonerius, ce qui ne signifie pas qu'il n'exerçait pas au même moment la charge de viceroi. Orefice 1982, p. 37, 58 l'identifie comme tel de 1424 à 1425. 
81 ASV, Cam. Ap., Intr. et Ex. 359, fo $102 \mathrm{r}^{\circ}$; ASV, Cam. Ap., Intr. et Ex. 360, fo $114 \mathrm{r}^{\circ}, 115 \mathrm{v}^{\circ}, 155 \mathrm{r}^{\circ}$; ASV, Instr. misc. 3242, $\mathrm{n}^{\circ} 44$; ASV, Instr. misc. 3337, $\mathrm{n}^{\circ} 13$; ASV, Reg. Aven. 254, f $259 \mathrm{v}^{\mathrm{o}}, 323 \mathrm{v}^{\circ}$; I diurnali del duca di Monteleone 1960, p. 48, 50-51; Jean Le Fèvre 1887, p. 222, 226-227, 269, 290, 293, 298, 306, 310, 314, 317-318.

82 Plusieurs des capitaines d'aventure servant Louis III furent ainsi, au moins partiellement si pas intégralement, stipendiés par le trésor de Martin V. Voir ASV, Cam. Ap., Intr. et Ex. 379, 382, 383, passim.

83 Les limites de cette virtualité se manifestent par exemple en 1392 lorsque le défi qui provoque la bataille de Barletta est adressé non au roi mais bien à son capitaine Ugo Sanseverino. Cela étant, la délégation de pouvoir n'est pas ignorée par les Duras puisque c'est précisément à l'homme désigné par le duc d'Anjou pour conduire ses armées que s'adresse leur cartel.

84 Faraglia 1894, p. 11.

85 Jean Le Fèvre 1887, p. 37 ; Barone 1887, p. 194 ; Cutolo 1977, p. 28 ; Labande 1939, p. 135.

86 Moscati 1936, p. 7-14.

87 Au point, en 1387, d'en investir Charles d'Anjou, frère cadet de Louis II, encore enfant. Jean Le Fèvre 1887 , p. 368.

88 Les exceptions existent toutefois, à l'image de Francesco dalla Ratta, comte de Caserta, fait connétable du royaume en 1394 selon le Chronicon Siculum 1887, p. 114.

89 Contamine 1972, p. 141-142.

90 Éd. dans Jarry 1896, p. 369-392.

91 Coville 1941, p. 10-41 et Avril 2009 ne mentionnent pas d'ouvrage de ce genre lorsqu'ils évoquent les figures de Louis ${ }^{\text {er }}$, Louis II et Louis III. Ce que l'on sait de la bibliothèque de René semble aller dans le même sens. Albanès 1875 ; Lecoy de La Marche 1873, p. 172-176, 261-263, 267-268.

92 Pour reprendre l'expression de Gautier 2009, p. 25.

93 Pour cette action, et plus largement sur la vie de Marie de Blois, voir Arlot 2006.

94 Plusieurs sont mentionnés dans ASV, Cam. Ap., Intr. et Ex. 337 et 356, passim.

95 Notons que Louis fut également encouragé à se concilier les barons calabrais par Jeanne II. Pontieri 1982, p. XXV.

96 Voir à ce sujet Bresc, Veauvy 1990, p. 83-84.

97 Contamine 1972, p. 541.

\section{Pour citer cet article}

Référence électronique

Christophe Masson, «Faire la guerre, faire l'État. Les officiers « militaires » sous les trois premiers souverains Valois de Naples », Mélanges de l'École française de Rome - Moyen Âge [En ligne], 127-1 I 2015, mis en ligne le 10 février 2015, consulté le 19 février 2015. URL : http:// mefrm.revues.org/2531

\section{À propos de l'auteur}

\section{Christophe Masson}

Université de Liège - «Transitions ». Département de recherches sur le Moyen Âge tardif \& la première Modernité - christophe.masson@ulg.ac.be

\section{Droits d'auteur}

C École française de Rome

\section{Résumés}

De Louis $\mathrm{I}^{\mathrm{er}}$ à René $\mathrm{I}^{\mathrm{er}}$, tous les ducs Valois d'Anjou tentèrent de prendre pied dans le royaume de Naples. Le premier d'entre eux avait en effet été adopté par la dernière reine de la dynastie capétienne - Jeanne $\mathrm{I}^{\mathrm{èr}}$. Organisant plusieurs expéditions militaires pour y établir 
leur pouvoir - contesté par les Anjou-Duras et ensuite les Aragon -, ces princes firent de leurs armées le cadre de leurs futures administrations. Nous verrons ici comment s'exerça le pouvoir militaire des trois premiers ducs d'Anjou. D'une part, les charges militaires à vocation géographique limitée furent le plus souvent confiés à des régnicoles tandis que, de l'autre, les officiers en charge de la conduite des armées en campagne ou des places intimement liées au prince (châteaux ou province de résidence) se révélèrent être, au contraire et sauf exception, des étrangers au royaume de Naples. En somme, ce fut le rapport de force entre les féodaux locaux et le duc d'Anjou qui dessina les structures militaires du pouvoir royal, chacun des deux partis y trouvant son intérêt.

From Louis $\mathrm{I}^{\text {st }}$ to René $\mathrm{I}^{\text {st }}$, the Valois dukes of Anjou tried to establish their power on the kingdom of Naples. The former had indeed been adopted by Jeanne $\mathrm{I}^{\text {st }}$, the last queen of the Capetian dynasty. The Valois dukes led several military expeditions to establish their power in Naples, facing the rivalry of the Anjou-Duras and then of the Aragon dynasty. These armies would later on constitute the framework of the Valois's local administration in Italy. This paper will examine how the three first dukes of Anjou exerted military power. On the one hand, the smaller military responsibilities — exerted on a limited geographical area-were most of the time entrusted to Neapolitean subjects. On the other hand, the officers in charge of the conduct of armies and of the strongholds close to the prince (castles or province of residence) turned out to be most of the time entrusted to foreigners. In sum, the balance of power between the local feudal lords and the duke of Anjou drew the military structures of the royal power; and each of the parties found there its own interest.

\section{Entrées d'index}

Mots-clés : Royaume de Naples, Ducs d'Anjou, Histoire militaire, Officiers Keywords : Kingdom of Naples, Dukes of Anjou, Military History, Officers

\section{Notes de l'auteur}

NDLA : Cet article s'inscrit dans le projet de recherche L'Europe angevine. Conception d'une base de données sur les officiers dans l'espace angevin (XIIIe-XVe siècles) et a pu être réalisé à l'occasion d'un séjour en tant que chercheur-résident de l'École française de Rome. Qu'il me soit ici permis de remercier les responsables du projet pour m'avoir donné l'opportunité d'y collaborer et l'équipe de cette institution pour l'accueil dont j'ai bénéficié en son sein. 